\title{
The degree of a $q$-holonomic sequence is a quadratic quasi-polynomial
}

\author{
Stavros Garoufalidis * \\ School of Mathematics \\ Georgia Institute of Technology \\ Atlanta, GA 30332-0160, USA \\ stavros@math.gatech.edu \\ http://www . math.gatech.edu/ stavros
}

Submitted: Jun 30, 2010; Accepted: Mar 5, 2011; Published: Mar 15, 2011

Mathematics Subject Classification: $05 \mathrm{C} 88$

\begin{abstract}
A sequence of rational functions in a variable $q$ is $q$-holonomic if it satisfies a linear recursion with coefficients polynomials in $q$ and $q^{n}$. We prove that the degree of a $q$-holonomic sequence is eventually a quadratic quasi-polynomial, and that the leading term satisfies a linear recursion relation with constant coefficients. Our proof uses differential Galois theory (adapting proofs regarding holonomic $D$ modules to the case of $q$-holonomic $D$-modules) combined with the Lech-MahlerSkolem theorem from number theory. En route, we use the Newton polygon of a linear $q$-difference equation, and introduce the notion of regular-singular $q$-difference equation and a WKB basis of solutions of a linear $q$-difference equation at $q=0$. We then use the Skolem-Mahler-Lech theorem to study the vanishing of their leading term. Unlike the case of $q=1$, there are no analytic problems regarding convergence of the WKB solutions. Our proofs are constructive, and they are illustrated by an explicit example.
\end{abstract}

\section{Contents}

1 Introduction $\quad 2$

1.1 History . . . . . . . . . . . . . . . . . . . . . 2

1.2 The degree and the leading term of a $q$-holonomic sequence . . . . . . . . . 3

1.3 The Newton polygon of a linear $q$-difference equation . . . . . . . . . . . . 4

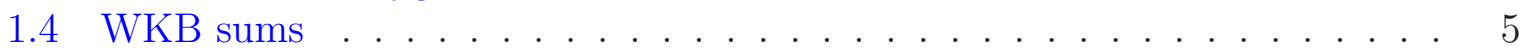

1.5 An example . . . . . . . . . . . . . . . . . . . . . . . 7

1.6 Plan of the paper . . . . . . . . . . . . . . . . . . 10

*To Doron Zeilberger, on the occasion of his 60th birthday 
2 Proof of Theorem $1.2 \quad 11$

2.1 Reduction to the case of a single slope . . . . . . . . . . . . . . . 11

2.2 Reduction to the case of a single eigenvalue . . . . . . . . . . . . . . . . . 12

2.3 First order linear $q$-difference equation . . . . . . . . . . . . . . . . . . 13

2.4 Proof of Theorem $1.2 \ldots \ldots \ldots \ldots \ldots$

2.5 The regular-singular non-resonant case . . . . . . . . . . . . . . . 14

3 Proof of Theorem 1.4 16

3.1 Generalized power sums $\ldots \ldots \ldots \ldots \ldots \ldots$

3.2 Proof of theorem $1.4 \ldots \ldots \ldots \ldots$. . . . . . . . . . . . . . . . . . . .

4 Invariants of $q$-holonomic sequences $\quad 18$

4.1 Synopsis of invariants . . . . . . . . . . . . . . . . 18

4.2 The annihilating polynomial of a $q$-holonomic sequence $\ldots \ldots \ldots . . .18$

4.3 The characteristic variety of a $q$-holonomic sequence . . . . . . . . . . . 19

4.4 The Newton polytope of a $q$-holonomic sequence . . . . . . . . . . . . . 19

4.5 The tropical curve of a $q$-holonomic sequence . . . . . . . . . . . . . . . 20

4.6 A tropical equation for the degree of a $q$-holonomic sequence . . . . . . . 20

4.7 Future directions . . . . . . . . . . . . . . . . . . 21

\section{Introduction}

\subsection{History}

$q$-holonomic sequences appear in abundance in Enumerative Combinatorics; [PWZ96, Sta97]. Here and and below, $q$ is a variable, and not a complex number. The fundamental theorem of Wilf-Zeilberger states that a multi-dimensional finite sum of a (proper) qhyper-geometric term is always $q$-holonomic; see [WZ92, Zei90, PWZ96]. Given this result, one can easily generate $q$-holonomic sequences. We learnt about this astonishing result from Doron Zeilberger in 2002. Putting this together with the fact that many statesum invariants in Quantum Topology are multi-dimensional sums of the above shape, it follows that Quantum Topology provides us with a plethora of $q$-holonomic sequences of natural origin; [GL05]. For example, the sequence of Jones polynomials of a knot and its parallels (technically, the colored Jones function) is $q$-holonomic. Moreover, the corresponding minimal recursion relation can be chosen canonically and is conjecturally related to geometric invariants of the knot; see [Gar04]. Recently, the author focused on the degree of a $q$-holonomic sequence, and in the case of the Jones polynomial it is also conjecturally related to topological invariants of knot complement; see [Gar11]. Since little is known about the Jones polynomial of a knot and its parallels, one might expect no regularity on its sequence of degree. The contrary is true, and in fact is a property of $q$-holonomic sequences and the focus of this paper. Our results were announced in [Gar11] and $[\mathrm{Gar}]$, where numerous examples of geometric/topological origin were discussed. 


\subsection{The degree and the leading term of a $q$-holonomic sequence}

Our main theorem concerns the degree and the leading term of a $q$-holonomic sequence. To phrase it, we need to recall what is a $q$-holonomic sequence, and what is a quasipolynomial. A sequence $\left(f_{n}(q)\right)$ of rational functions is $q$-holonomic if it satisfies a recursion relation of the form

$$
a_{d}\left(q^{n}, q\right) f_{n+d}(q)+\cdots+a_{0}\left(q^{n}, q\right) f_{n}(q)=0
$$

for all $n$ where $a_{j}(u, v) \in \mathbb{Q}[u, v]$ for $j=0, \ldots, d$ and $a_{d}(u, v) \neq 0$.

Given a polynomial with rational coefficients

$$
f(q)=\sum_{m}^{M} c_{i} q^{i} \in \mathbb{Q}[q]
$$

that satisfies $c_{m} c_{M} \neq 0$, its degree (also known as the order in different contexts) $\delta(f(q)$ ) and its leading term $\operatorname{lt}(f(q))$ is given by $m$ and $c_{m}$ respectively. In other words, the degree and the leading term of $f(q)$ is the order and the starting coefficient in the Taylor series expansion of $f(q)$ at $q=0$. The degree and leading term can be uniquely extended to the field $\mathbb{Q}(q)$ of all rational functions, the ring $\mathbb{Q}[[q]]$ of formal power series in $q$, the field $\mathbb{Q}((q))$ of all Laurent series in $q$ and finally to the algebraically closed field

$$
K=\overline{\mathbb{Q}}\{\{q\}\}=\cup_{r=1}^{\infty} \overline{\mathbb{Q}}\left(\left(q^{1 / r}\right)\right)
$$

of all Puiseux series in $q$ with algebraic coefficients (see [Wal78]). The field $K$ is required in Theorems 1.2 and 1.3 below.

Recall that a quasi-polynomial $p(n)$ is a function

$$
p: \mathbb{N} \longrightarrow \mathbb{N}, \quad p(n)=\sum_{j=0}^{d} c_{j}(n) n^{j}
$$

for some $d \in \mathbb{N}$ where $c_{j}(n)$ is a periodic fuction with integral period for $j=1, \ldots, d$; [Sta97, BR07]. If $c_{j}=0$ for $j>2$, then we will say that $p$ is a quadratic quasi-polynomial. We will say that a function is eventually equal to a quasi-polynomial if they agree for all but finitely many values.

Theorem 1.1. (a) The degree of a $q$-holonomic sequence is eventually a quadratic quasipolynomial.

(b) The leading term of a $q$-holonomic sequence eventually satisfies a linear recursion with constant coefficients.

Theorem 1.1 follows from Theorems 1.2 and 1.4 below.

Remark 1.1. If $f_{n}(q) \in \mathbb{Z}\left[q^{ \pm 1}\right]$ is $q$-holonomic with degree $\delta_{n}$ and leading term $\mathrm{lt}_{n}$, it follows that $f_{n}(q)-\mathrm{lt}_{n} q^{\delta_{n}}$ is also $q$-holonomic. Thus, Theorem 1.1 applies to each one of the terms of $f_{n}(q)$. 
Remark 1.2. L. Di Vizio brought to our attention that results similar to part (a) of Theorem 1.1 appear in [BB92, Thm.4.1] and also in [DV08, Sec.1.1,Sec.1.3]. However, the statement and proof of Theorem 1.1 appear to be new.

Our next corollary to Theorem 1.1 characterizes which sequences of monomials are $q$-holonomic sequences. In a sense, such sequences are building blocks of all $q$-holonomic sequences.

Corollary 1.3. If $\left(a_{n}\right)$ and $\left(b_{n}\right)$ are sequences of integers (with $a_{n} \neq 0$ for all $n$ ), then $\left(a_{n} q^{b_{n}}\right)$ is $q$-holonomic if and only if $b_{n}$ is holonomic and $a_{n}$ is a quadratic quasi-polynomial for all but finitely many $n$.

\subsection{The Newton polygon of a linear $q$-difference equation}

As is common, we can write a linear $q$-difference equation (1) in operator form by introducing two operators $L$ and $M$ which act on a sequence $\left(f_{n}(q)\right)$ by

$$
(L f)_{n}(q)=f_{n+1}(q), \quad(M f)_{n}(q)=q^{n} f_{n}(q) .
$$

It is easy to see that the operators $M$ and $L q$-commute

$$
L M=q M L
$$

and generate the so-called $q$-Weyl algebra

$$
\mathcal{D}=\cup_{r=1}^{\infty} K\left(\left(M^{1 / r}\right)\right)\langle L\rangle /\left(L M^{1 / r}-q^{1 / r} M L\right) .
$$

We will call an element of $\mathcal{D}$ a linear q-difference operator. The general element of $\mathcal{D}$ is of the form

$$
P=\sum_{i=0}^{d} a_{i}(M, q) L^{i}
$$

where $a_{i}(M, q) \in K\left(\left(M^{1 / r}\right)\right)$ for $i=0, \ldots, d$ and $a_{d}(M, q) \neq 0$. The equation

$$
P f=0
$$

for a $K$-valued sequence $f_{n}(q)$ is exactly the recursion relation (1). The Newton polygon $N(P)$ of an element $P \in \mathcal{D}$ is defined to be the lower convex hull of the points $\left(i, \delta_{M}\left(a_{i}\right)\right)$ where $\delta_{M}$ denotes the smallest degree with respect to $M$. Note that usually the Newton polygon $N^{\prime}(P)$ of a 2-variable polynomial is defined to be the convex hull of the exponents of its monomials. Since we are working locally at $q=0$, we view $N^{\prime}(P)$ by placing our eye at $-\infty$ in the vertical axis and looking up. The resulting object is the lower convex hull $N(P)$ defined above. The Newton polygon of a linear $q$-difference equation was also studied in the recent Ph.D. thesis by P. Horn; see [Hor09, Chpt.2].

The Newton polygon $N(P)$ of $P$ is a finite union of intervals with rational end points and two vertical rays. Each interval with end-points $\left(i, d_{i}\right)$ and $\left(j, d_{j}\right)$ for $i<j$ has a 
slope $s=\left(d_{j}-d_{i}\right) /(j-i)$ and a length (or multiplicity) $l=j-i$. The multiset of slopes $s(N(P))$ of $N(P)$ is the set of slopes of $s N(P)$ each with multiplicity $l_{s}$. An example of a Newton polygon of a linear $q$-difference operator of degree 7 with three slopes $-1,0,1 / 2$ of multiplicity 2, 1, 4 respectively is shown here:

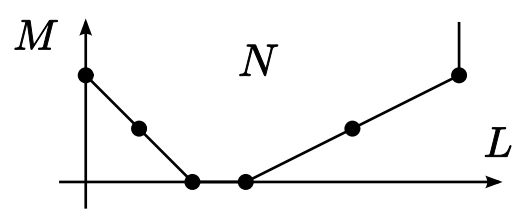

The Newton polygon is a convenient way to organize solutions to a linear $q$-difference equation. The reader may compare this with the Newton polygon of a linear differential operator attributed to Malgrange and Ramis; see [vdPS03, Sec.3.3] and references therein. In analogy with the theory of linear differential operators, we will say that $P$ is a regularsingular q-difference operator if its Newton polygon consists of a single horizontal segment. In other words, after a minor change of variables, with the notation of (5) this means that $a_{i}(M, q) \in K\left[\left[M^{1 / r}\right]\right]$ for all $i$ and $a_{0}(0, q) a_{d}(0, q) \neq 0$.

\subsection{WKB sums}

Since Equation (1) involves two independent variables $q$ and $q^{n}$, let us set $q^{n}=u$ and consider the $q$-difference equation

$$
a_{d}(u, q) f\left(u q^{d}, q\right)+\cdots+a_{0}(u, q) f(u, q)=0
$$

Note that if $f(u, q)$ solves $(6)$, and if $f_{n}(q):=f\left(q^{n}, q\right)$ makes sense, then $f_{n}(q)$ solves (1) and vice-versa. It turns out that the general solution to (6) is a WKB sum. The latter are generalizations of the better known generalized power sums discussed in Section 3.1 below.

Definition 1.4. (a) A formal WKB series is an expression of the form

$$
f_{\tau}(u, q)=q^{\gamma n^{2}} \lambda(q)^{n} A(n, u, q)
$$

where $\tau=(\gamma, \lambda(q), A)$ and

- the exponent $\gamma$ is a rational number,

$$
A(n, u, q)=\sum_{i=0}^{M} \sum_{k=0}^{\infty} \phi_{i, k}(q) u^{k / r} n^{i}
$$

is a polynomial in $n$ with coefficients in $\overline{\mathbb{Q}}\left(\left(q^{1 / r}\right)\right)\left[\left[u^{1 / r}\right]\right]$ for some $r \in \mathbb{N}$.

- $\lambda(q) \in \overline{\mathbb{Q}}\left(\left(q^{1 / r}\right)\right)$ and $\phi_{0,0}(q)=1$.

- there exists $c \in \mathbb{Q}$ so that $\delta\left(\phi_{i, k}(q)\right) \geq c k$ for all $i$ and $k$ 
(b) A formal WKB sum is a finite $K$-linear combination of formal WKB series.

Observe that if $f_{\tau}(u, q)$ is a formal WKB series, then its evaluation

$$
f_{\tau, n}(q)=f_{\tau}\left(q^{n}, q\right) \in K
$$

is a well-defined $K$-valued sequence for $n>-r c$.

Observe further if $f_{\tau}(u, q)$ is given by $(7)$, the operators $L$ and $M$ act on $f_{\tau}(u, q)$ by:

$$
\left(L f_{\tau}\right)(u, q)=q^{\gamma(n+1)^{2}} \lambda(q)^{n+1} A(n+1, u q, q), \quad\left(M f_{\tau}\right)(u, q)=q^{\gamma n^{2}} \lambda(q)^{n} u A(n, u, q) .
$$

Theorem 1.2. Every linear q-difference equation has a basis of solutions of the form $f_{\tau, n}(q)$. Moreover, the multiset of exponents of the bases is the multiset of the negatives of the slopes of the Newton polygon.

Recall that $K\{\{M\}\}$ denotes the field of Puiseux series in a variable $M$ with coefficients in $K$. Let $\delta_{M}(f)$ denote the minimum exponent of $M$ in a Puiseux series $f \in K\{\{M\}\}$.

Theorem 1.3. Every monic q-difference operator $P \in \mathcal{D}$ of order $d$ can be factored as an ordered product

$$
P=\prod_{i=1}^{d}\left(L-a_{i}(M, q)\right)
$$

where $a_{i}(M, q) \in K\{\{M\}\}$ and the multiset $\left\{\delta_{M}\left(a_{i}\right) \mid i=1, \ldots, d\right\}$ of slopes is the negative of the multiset of slopes of the Newton polygon of $P$.

Remark 1.5. The WKB expansion of solution of linear $q$-difference equations given in Theorem 1.2 appears to be new, and perhaps it is related to some recent work of Witten [Wit], who proposes a categorification of the colored Jones polynomial in an arbitrary 3-manifold. The curious reader may compare [Wit, Eqn.6.21] with our Theorem 1.2. We wish to thank T. Dimofte for pointing out the reference to us.

Remark 1.6. Although the proofs of Theorems 1.2 and 1.3 follow the well-studied case of linear differential operators in one variable $x$, we are unable to formulate an analogue of Theorems 1.2 and 1.3 in the differential operator case. Indeed, $q$ is a variable which seems to be independent of the spacial variables $x$ of a differential operator. The meaning of the variable $q$ can be explained by Quantization, or from Tropical Geometry (where it is usually denoted by $t$ ) or from the representation theory of Quantum Groups; see for example [Gar] and referencies therein, and also Section 4 below.

Theorem 1.4. If $f_{n}(q)$ is a finite $K$-linear combination of formal WKB series of the form (7), then for large $n$ its degree is given by a quadratic quasi-polynomial and its leading term satisfies a linear recursion relation with constant coefficients. 


\subsection{An example}

In this section we discuss in detail an example to illustrate the introduced notions and the content of Theorems 1.1, 1.2 and 1.4. The example shows that the proof of Theorem 1.1 and the WKB expansion of Theorem 1.4 is algorithmic, despite the use of differential Galois theory. Consider the $q$-holonomic sequence $f_{n}(q) \in \mathbb{Z}[q]$ whose first few terms are given by:

$$
\begin{aligned}
f_{0}= & 1 \\
f_{1}= & 2-q^{2} \\
f_{2}= & 3+q-2 q^{3}-2 q^{4}+q^{7} \\
f_{3}= & 4+2 q+2 q^{2}-3 q^{4}-4 q^{5}-4 q^{6}-q^{7}+2 q^{9}+2 q^{10}+2 q^{11}-q^{15} \\
f_{4}= & 5+3 q+4 q^{2}+3 q^{3}+q^{4}-4 q^{5}-6 q^{6}-8 q^{7}-8 q^{8}-4 q^{9}-2 q^{10}+3 q^{11}+4 q^{12}+7 q^{13} \\
& +5 q^{14}+4 q^{15}+q^{16}-2 q^{18}-2 q^{19}-2 q^{20}-2 q^{21}+q^{26} \\
f_{5}= & 6+4 q+6 q^{2}+6 q^{3}+6 q^{4}+2 q^{5}-3 q^{6}-8 q^{7}-12 q^{8}-15 q^{9}-16 q^{10}-11 q^{11}-8 q^{12}+5 q^{14} \\
& +12 q^{15}+14 q^{16}+16 q^{17}+12 q^{18}+10 q^{19}+4 q^{20}-q^{21}-4 q^{22}-7 q^{23}-8 q^{24}-8 q^{25}-5 q^{26} \\
& -4 q^{27}-q^{28}+2 q^{30}+2 q^{31}+2 q^{32}+2 q^{33}+2 q^{34}-q^{40} \\
f_{6}= & 7+5 q+8 q^{2}+9 q^{3}+11 q^{4}+9 q^{5}+7 q^{6}-2 q^{7}-7 q^{8}-15 q^{9}-22 q^{10}-28 q^{11}-30 q^{12} \\
& -26 q^{13}-22 q^{14}-11 q^{15}-2 q^{16}+13 q^{17}+21 q^{18}+33 q^{19}+34 q^{20}+36 q^{21}+30 q^{22}+25 q^{23} \\
& +11 q^{24}+3 q^{25}-8 q^{26}-17 q^{27}-22 q^{28}-24 q^{29}-24 q^{30}-20 q^{31}-14 q^{32}-10 q^{33}-q^{34} \\
& +2 q^{35}+7 q^{36}+8 q^{37}+11 q^{38}+9 q^{39}+8 q^{40}+5 q^{41}+4 q^{42}+q^{43}-2 q^{45}-2 q^{46}-2 q^{47} \\
& -2 q^{48}-2 q^{49}-2 q^{50}+q^{57}
\end{aligned}
$$

The general term of the sequence $f$ is given by:

$$
f_{n}(q)=\sum_{k=0}^{n} \frac{(q)_{n+k}}{(q)_{n-k}(q)_{k}}
$$

where the $q$-factorial is defined by

$$
(q)_{n}=\prod_{k=1}^{n}\left(1-q^{k}\right), \quad(q)_{0}=1 .
$$

The above expression implies via the Wilf-Zeilberger theorem that $f$ is $q$-holonomic; see [WZ92]. The qzeil.m implementation of the Wilf-Zeilberger proof developed by [PR97, PR] gives that $f$ is annihilated by the following operator:

$$
\begin{aligned}
P_{f}= & \left(-1+M q^{2}\right)\left(-1+M q+M^{2} q^{2}\right) L^{2}+\left(-2+M q+M q^{2}+2 M^{2} q^{2}+M^{2} q^{3}+2 M^{2} q^{4}\right. \\
& \left.-2 M^{3} q^{4}-2 M^{3} q^{5}-M^{4} q^{5}-M^{4} q^{6}-M^{4} q^{7}+M^{5} q^{7}+M^{5} q^{8}+M^{6} q^{9}\right) L \\
& +1-M q^{2}-M^{2} q^{4} .
\end{aligned}
$$


The 2-dimensional Newton polytope $\mathcal{N}_{2}\left(P_{f}\right)$ and the Newton polygon $N\left(P_{f}\right)$ are given by:
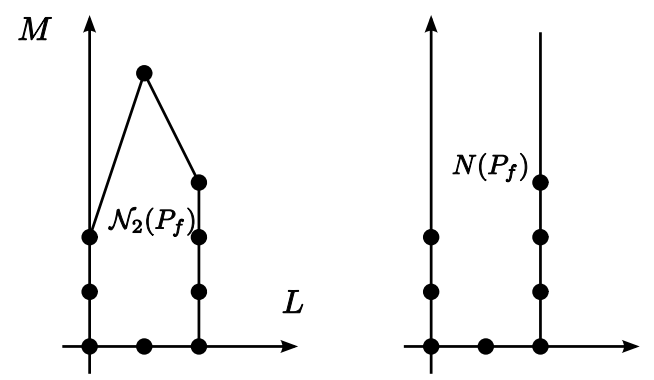

$P_{f}$ is a second order regular-singular $q$-difference operator. Its Newton polygon $N\left(P_{f}\right)$ has only one slope 0 with multiplicity 2 . The edge polynomial of the 0 -slope is cyclotomic $(L-1)^{2}$ with two equal roots (i.e., eigenvalues) 1 and 1 . The degree $\delta_{n}$ and the leading term $\operatorname{lt}_{n}$ of $f_{n}(q)$ are given by $\delta_{n}=0$ and $l_{n}=n+1$ for all $n$.

The characteristic curve $\operatorname{ch}_{f}$ (discussed in Section 4) is reducible given by the zeros $(L, M) \in$ $\left(\mathbb{C}^{*}\right)^{2}$ of the polynomial

$$
\left(-1+M+M^{2}\right)\left(-1+2 L-L^{2}+L^{2} M-3 L M^{2}+L M^{3}+L M^{4}\right)=0
$$

The tropical.lib program of [Mar] computes the vertices of the tropical curve $\mathcal{T}_{f}$ are:

$$
(-1,-2), \quad(3,-2), \quad(0,-3 / 2), \quad(1,-3 / 2), \quad(0,-1)
$$

The next figure is a drawing of the tropical curve $\mathcal{T}_{f}$ and its multiplicities, where edges not labeled have multiplicity 1.

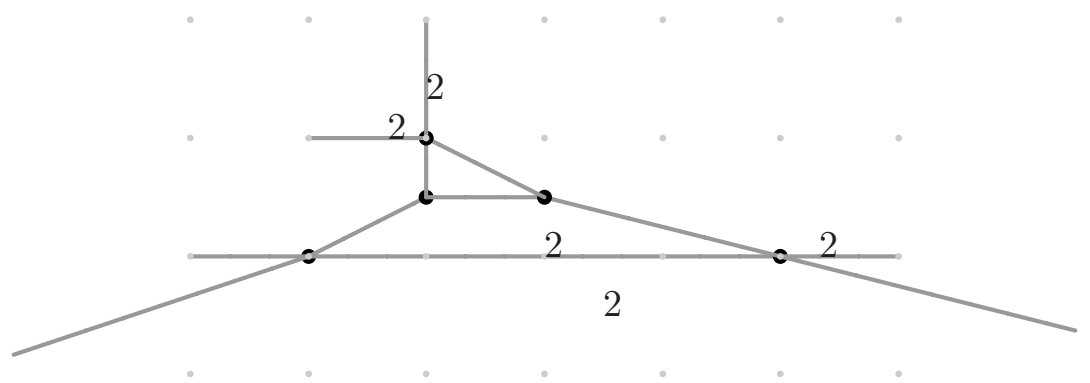

The Newton subdivision of the Newton polytope $\mathcal{N}_{2}\left(P_{f}\right)$ is:

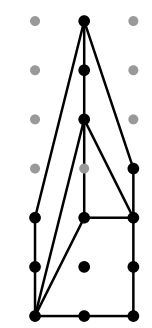


To illustrate Theorem 1.1, observe that $f_{n}(q)$ is a sequence of Laurent polynomials. The minimum (resp., maximum) degree $\delta_{n}$ (resp., $\hat{\delta}_{n}$ ) of $f_{n}(q)$ with respect to $q$ is given by:

$$
\delta_{n}=0, \quad \hat{\delta}_{n}=\frac{n(3 n+1)}{2}
$$

The coefficient $\mathrm{lt}_{n}$ (resp., $\widehat{\mathrm{lt}}_{n}$ ) of $q^{\delta_{n}}$ (resp., $q^{\hat{\delta}_{n}}$ ) in $f_{n}(q)$ is given by:

$$
\mathrm{lt}_{n}=n+1, \quad \widehat{\mathrm{lt}}_{n}=(-1)^{n}
$$

$\mathrm{lt}_{n}$ and $\widehat{\mathrm{lt}}_{n}$ are holonomic sequences that satisfy linear recursion relations with constant coefficients.

To illustrate Theorem 1.4, observe that there is a single 0 slope of length 2 with edge polynomial $(L-1)^{2}$ and eigenvalue 1 with multiplicity 2 . This is a resonant case. Theorem 1.4 dictates that we we substitute the WKB ansatz

$$
\hat{f}_{n+j}(q)=\sum_{k=0}^{\infty}\left(\phi_{k}(q)+(n+j) \psi_{k}(q)\right) q^{(n+j) k}
$$

in the recursion $P_{f} \hat{f}=0$, collect terms with respect to $M=q^{k}$ and with respect to $n$ and set them all equal to zero. It follows that the vector $\left(\psi_{k}(q), \phi_{k}(q)\right)$ satisfies a sixth order linear recursion relation:

$$
\begin{aligned}
\psi_{k}= & \frac{1}{\left(1-q^{k}\right)^{2}}\left\{q^{3+k} \psi_{-6+k} q^{2+k}(1+q) \psi_{-5+k}+q^{1+k}\left(1+q+q^{2}\right) \psi_{-4+k}\right. \\
& +q^{-2+k}\left(2 q^{3}+2 q^{4}-q^{k}\right) \psi_{-3+k}-\left(-q^{4}-q^{2 k-2}+2 q^{k}+q^{1+k}+2 q^{2+k}+q^{-1+2 k}\right) \psi_{-2+k} \\
& \left.+\left(q^{2}+q^{2 k-1}-q^{k}-q^{1+k}+q^{2 k}\right) \psi_{-1+k}\right\} \\
\phi_{k}= & \frac{1}{\left(1-q^{k}\right)^{2}}\left\{-q^{3+k} \phi_{-6+k}-q^{2+k}(1+q) \phi_{-5+k}+q^{1+k}\left(1+q+q^{2}\right) \phi_{-4+k}\right. \\
& +q^{-2+k}\left(2 q^{3}+2 q^{4}-q^{k}\right) \phi_{-3+k}-\frac{\left(-q^{6}-q^{2 k}+2 q^{2+k}+q^{3+k}+2 q^{4+k}+q^{1+2 k}\right) \phi_{-2+k}}{q^{2}} \\
& +\frac{\left(q^{3}+q^{2 k}-q^{1+k}-q^{2+k}+q^{1+2 k}\right) \phi_{-1+k}+\frac{q^{3+k}\left(1+q^{k}\right) \psi_{-6+k}}{\left(-1+q^{k}\right)}}{\left(-1+q^{k}\right)} \\
& +\frac{q^{2+k}(1+q)\left(1+q^{k}\right) \psi_{-5+k}-\frac{q^{1+k}\left(1+q+q^{2}\right)\left(1+q^{k}\right) \psi_{-4+k}}{\left(-1+q^{k}\right)}}{\left(-1+q^{k}\right)} \\
& -\frac{2 q^{-2+k}\left(q^{3}+q^{4}-q^{k}+q^{3+k}+q^{4+k}\right) \psi_{-3+k}}{\left(-1+q^{k}\right)} \\
& +\frac{q^{-2+k}\left(2 q^{2}+q^{3}+2 q^{4}-2 q^{6}-2 q^{k}+2 q^{1+k}+2 q^{2+k}+q^{3+k}+2 q^{4+k}\right) \psi_{-2+k}}{\left(-1+q^{k}\right)} \\
& +\frac{q^{-1+k}\left(q+q^{2}-2 q^{3}-2 q^{k}-q^{1+k}+q^{2+k}\right) \psi_{-1+k}}{(1+1)}
\end{aligned}
$$

with an arbitrary initial condition $\left(\psi_{0}, \phi_{0}\right) \in \mathbb{Q}[[q]]^{2}$. Note that $\psi_{k}$ the first equation above is a recursion relation for $\psi_{k}$ and the second equation is a recursion for $\phi_{k}$ that also involves $\psi_{k^{\prime}}$ 
for $k^{\prime}<k$. This is a general feature of the WKB in the case of resonanse. It follows that our particular solution (11) of the $q$-difference equation $P_{f} f=0$ has the form:

$$
f_{n}(q)=\sum_{k=0}^{\infty}\left(\phi_{k}(q)+n \psi_{k}(q)\right) q^{n k} \in \mathbb{Q}[[q]]
$$

where $\left(\psi_{k}, \phi_{k}\right)$ are defined the above recursion with suitable initial conditions. Note that Equation (12) implies that the coefficient $c_{m, n}=a_{m}+n b_{m}$ of $q^{m}$ in $f_{n}(q)$ is a linear function of $n$ for $n>m$. These values determine our initial conditions by:

$$
\phi_{0}(q)=\sum_{m=0}^{\infty} a_{m} q^{m}
$$

and

$$
\psi_{0}(q)=\sum_{m=0}^{\infty} b_{m} q^{m}
$$

In fact, a direct computation shows that

$$
\begin{aligned}
\phi_{0}(q)= & 1-q-4 q^{2}-9 q^{3}-19 q^{4}-33 q^{5}-59 q^{6}-93 q^{7}-150 q^{8}-226 q^{9}-342 q^{10}-494 q^{11} \\
& -721 q^{12}-1011 q^{13}-1425 q^{14}-1960 q^{15}-2695 q^{16}-3633 q^{17}-4903 q^{18}-6506 q^{19} \\
& -8633 q^{20}-11312 q^{21}-14796 q^{22}-19157 q^{23}-24773 q^{24}-31744 q^{25}-40608 q^{26} \\
& -51578 q^{27}-65372 q^{28}-82341 q^{29}-103522 q^{30}+O\left(q^{31}\right)
\end{aligned}
$$

and

$$
\begin{aligned}
\psi_{0}(q)= & 1+q+2 q^{2}+3 q^{3}+5 q^{4}+7 q^{5}+11 q^{6}+15 q^{7}+22 q^{8}+30 q^{9}+42 q^{10}+56 q^{11}+77 q^{12} \\
& +101 q^{13}+135 q^{14}+176 q^{15}+231 q^{16}+297 q^{17}+385 q^{18}+490 q^{19}+627 q^{20}+792 q^{21} \\
& +1002 q^{22}+1255 q^{23}+1575 q^{24}+1958 q^{25}+2436 q^{26}+3010 q^{27}+3718 q^{28}+4565 q^{29} \\
& +5604 q^{30}+O\left(q^{31}\right)
\end{aligned}
$$

The reader may recognize (and also confirm by an explicit computation) that

$$
\psi_{0}(q)=\frac{1}{(q ; q)_{\infty}}=\prod_{m=0}^{\infty} \frac{1}{1-q^{m}}
$$

An extra-credit problem is to give an explicit formula for the power series $\phi_{0}(q)$. Note finally that Equation (11) implies that the specialization of $f_{n}(q)$ at $q=1$ is given by:

$$
f_{n}(1)=1
$$

for all $n$, much like the case of the colored Jones polynomial of a knot, [GL05].

\subsection{Plan of the paper}

Theorem 1.1 follows from Theorems 1.2 and 1.4.

Theorem 1.2 follows by two reductions using a $q$-analogue of Hensel's lemma, analogous to the case of linear differential operators. The first reduction factors an operator with arbitrary 
Newton polygon into a product of operators with a Newton polygon with a single slope. After a change of variables, we can assume that these operators are regular-singular. The second reduction factors a regular-singular $q$-difference operator into a product of first order regularsingular operators with eigenvalues of possibly equal constant term. Thus, we may assume that the $q$-difference operator is regular-singular with eigenvalues of equal constant term. In that case, we can construct a basis of formal WKB solutions of the required type. The above proof also factorizes a linear $q$-difference operator into a product of first order $q$-difference operators, giving a proof of Theorem 1.3.

Theorem 1.4 follows from the Lech-Mahler-Skolem theorem on the zeros of generalized exponential sums.

Finally, in Section 4, which is independent of the results of the paper, we discuss several invariants of $q$-holonomic sequences, and compute them all in some simple examples.

\section{Proof of Theorem 1.2}

\subsection{Reduction to the case of a single slope}

Theorem 1.2 follows ideas similar to the case of linear differential operators, presented for example in [vdPS03, Sec.3]. Rather than develop the whole theory, we will highlight the differences between the differential case and the $q$-difference case.

In the case of linear differential operators, the basic operators $x$ and $x \partial_{x}$ satisfy the inhomogeneous commutation relation

$$
\left[x \partial_{x}, x\right]=x
$$

The right hand side of the above commutation relation leads to consider Newton polygons are convex hulls of translates of the second quadrant $\mathbb{R}_{-} \times \mathbb{R}^{+}$. For examples of such polygons, see [vdPS03, Sec.3.3]. As a result, the slopes of the Newton polygons of linear differential operators are always non-negative rational numbers. On the other hand, in the $q$-difference case, the $q$-commutation relation (3) preserves the $L$ and $M$ degree of a monomial in $M, L$.

In the case of linear differential operators of a single variable $x$, the WKB solutions involve power series in $x^{1 / r}$ and polynomials in $\log x$. In the case of linear $q$-difference operators, the WKB solutions involve power series in $q^{n}$ and polynomials in $n$. In addition, the convergence conditions are easier to deal with when $q=0$, and much harder when $q=1$. Convergence when $q=1$ involves regularizing factorially divergent formal power series.

Consider a $q$-difference operator $P$ and its Newton polygon $N(P)$. In this section, we will allow the coefficients $a_{i}(M, q)$ of $P$ from Equation (5) to be arbitrary elements of the field $K\{\{M\}\}$. Recall that the Newton polygon $N(P)$ consists of finitely many segments and two rays. We will say that $P$ is slim if all its monomials lie in the bounded segments of its Newton

polygon. We will say that $P_{1}>P_{2}$ if the boundary of $N\left(P_{1}\right)$ lies in the interior of $P_{2}$ or in the interior of the two rays of $P_{2}$. We will say that an operator $P$ from Equation (5) is monic if $a_{d}(M, q)=1$. Recall the Minkowski sum

$$
N_{1}+N_{2}=\left\{a+b \mid a \in N_{1}, b \in N_{2}\right\}
$$

of two polytopes in the plane. Here and below, we will always consider the Minkowski sum of two convex polytopes that arise from linear $q$-difference operators, i.e., polytopes that consist of two vertical rays and some bounded segments. 
The next lemma is a $q$-version of Hensel's lifting lemma.

Lemma 2.1. If $P$ is monic and slim, for every partition of its set of slopes in two different sets $S_{1}$ and $S_{2}$ there exist unique slim and monic operators $P_{1}$ and $P_{2}$ with slopes $S_{1}$ and $S_{2}$ respectively, and an operator $R(P)$ so that

$$
P=P_{1} P_{2}+R(P), \quad R(P)>P .
$$

Proof: This follows as in the differential case. The only difference is that monomials $M^{a} L^{b}$ and $M^{a^{\prime}} L^{b^{\prime}}$ commute up to a power of $q$. The next example will explain the proof. Suppose $P=a M+b L+c M^{2} L+M^{3} L^{2}$ is a monic slim operator with Newton polygon $N$ with two slopes, where $a, b, c \in K$. Now $N=N_{1}+N_{2}$ shown as follows:

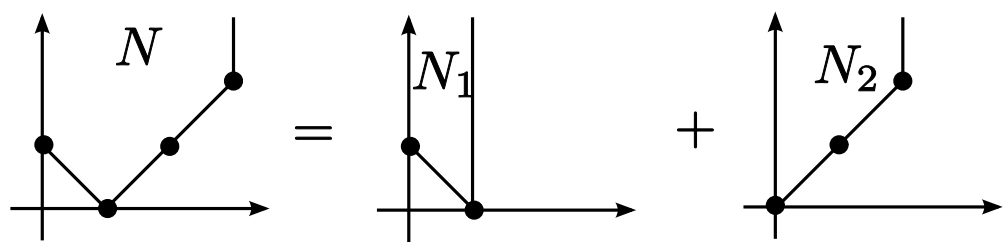

We want to find unique $x_{0}, y_{0}, y_{1}, y_{2} \in K$ so that

$$
a M+b L+c M^{2} L+M^{3} L^{2}=\left(x_{0} M+L\right)\left(y_{0}+y_{1} M L+y_{2} M^{2} L^{2}\right) .
$$

Multiplying out, we obtain that

$$
\begin{aligned}
a M+b L+c M^{2} L+M^{3} L^{2} & =x_{0} y_{0} M+y_{0} L+y_{1} L M L+R(P) \\
R(P) & =x_{0} y_{1} M^{2} L+x_{0} M^{3} L^{2}+L M^{2} L^{2}>P
\end{aligned}
$$

Thus, we obtain the system of equations

$$
x_{0} y_{0}=a, \quad y_{0}=b, \quad q y_{1}=c .
$$

The above system can be solved uniquely in $K$ whether $q$ is present or not.

Proposition 2.2. Suppose $P$ is monic and $N(P)=N_{1}+N_{2}$ where $N_{1}$ and $N_{2}$ have no slope in common. Then there exist unique monic operators $P_{1}$ and $P_{2}$ with Newton polygons $N_{1}$ and $N_{2}$ such that $P=P_{1} P_{2}$. In addition, we have:

$$
\mathcal{D} / \mathcal{D} P=\mathcal{D} / \mathcal{D} P_{1} \oplus \mathcal{D} / \mathcal{D} P_{2}
$$

Proof: It follows verbatim from the proof of Theorem 3.48 in [vdPS03] using Lemma 2.1.

\subsection{Reduction to the case of a single eigenvalue}

Suppose that $P$ is a linear $q$-difference operator with a single slope. After a change of variables $f_{n}(q)=q^{n^{2} \gamma+n \eta} g_{n}(q)$, we can assume that the slope is zero, i.e. that $P$ is regular singular. In other words, if $P$ is given by $(6)$, then $a_{d}(0, q) a_{0}(0, q) \neq 0$. Then, the slim part $R(P)$ of $P$ is given by

$$
R(P)=\sum_{i=0}^{d} a_{i}(0, q) L^{i}
$$

where $P-R(P)>P$. We may think of $R(P)$ as a polynomial in a variable $L$ with coefficients in the field $K$. Suppose that $R(P)$ is monic and we can factor $R(P)=A B$ as the product of two relatively prime monic polynomials. 
Proposition 2.3. With the above assumptions there exist unique monic regular-singular operators $P_{1}$ and $P_{2}$ such that $P=P_{1} P_{2}$ and $R\left(P_{1}\right)=A$ and $R\left(P_{2}\right)=B$. Moreover,

$$
\mathcal{D} / \mathcal{D} P=\mathcal{D} / \mathcal{D} P_{1} \oplus \mathcal{D} / \mathcal{D} P_{2} .
$$

Proof: It follows verbatim from the proof of Proposition 3.50 in [vdPS03] using Lemma 2.1.

\subsection{First order linear $q$-difference equation}

In this section we will prove Theorem 1.2 for the first order linear $q$-difference equation

$$
f_{n+1}(q)-b\left(q^{n}, q\right) f_{n}(q)=0
$$

where $b(M, q) \in K((M))$. If $b(M, q)=M^{\gamma} c(M, q)$ where $c(M, q) \in K[[M]]$ and $c(0, q) \neq 0$ the substitution $f_{n}(q)=q^{n^{2} \gamma / 2} c(0, q)^{n} g_{n}(q)$ gives the equation

$$
g_{n+1}(q)-a\left(q^{n}, q\right) g_{n}(q)=0
$$

where $a(M, q)=q^{\gamma / 2} \frac{c(M, q)}{c(0, q)} \in K[[M]]$ satisfies $a(0, q)=1$. A solution to Equation (14) is

$$
\begin{aligned}
f_{n}(q) & =a(q, q) a\left(q^{2}, q\right) \ldots a\left(q^{n-1}, q\right) \\
& =\frac{\prod_{k=1}^{\infty} a\left(q^{k}, q\right)}{a\left(q^{n}, q\right) a\left(q^{n+1}, q\right) \ldots}
\end{aligned}
$$

Now

$$
a(M, q)=1-\sum_{k=1}^{\infty} M^{k} a_{k}(q)
$$

where $a_{k}(q) \in K$, implies that

$$
\frac{1}{a(M, q)}=1+\sum_{l=1}^{\infty}\left(\sum_{k=1}^{\infty} M^{k} a_{k}(q)\right)^{l} \in u K[[u]]
$$

Consequently,

$$
f(M, q)=\frac{1}{\prod_{i=0}^{\infty} a\left(M q^{i}, q\right)} \in K[[M]]
$$

Let us now match this solution with a WKB sum. Consider

$$
f(u, q)=\sum_{k=0}^{\infty} \phi_{k}(q) u^{k} \in K[[u]]
$$

where $\phi_{0}=1$ and substitute into the equation

$$
f(u q, q)-a(u, q) f(u, q)=0
$$

we obtain an equation in the ring $K[[u]]$. The vanishing of the coefficient of $u^{k}$ gives

$$
\phi_{k}=\frac{1}{1-q^{k}} \sum_{i=1}^{k} a_{i} \phi_{k-i}
$$

Thus the WKB solution matches exactly the solution (15) for the first-order regular singular equation (14). 


\subsection{Proof of Theorem 1.2}

Propositions 2.2 and 2.3 and a possible replacement of $q$ by $q^{1 / r}$ reduce Theorem 1.2 to the case of a monic regular-singular differential operator of the form

$$
P=\prod_{i=1}^{d}\left(L-a_{i}(M, q)\right)
$$

where $a_{i}(M, q) \in K[[M]]$ for all $i$ and $a(0, q)=1$. It suffices to find a basis of solutions of the equation $P f=0$ of the form

$$
f(u, q)=\sum_{k=0}^{\infty} \phi_{k}(n, q) u^{k}
$$

where $\phi_{k}(n, q) \in K[n]$ are polynomials of degree at most $d$. We will use induction on $d$. When $d=1$, this is proven in Section 2.3. Let $Q=\prod_{i=2}^{d}\left(L-a_{i}(M, q)\right)$. If we set $g_{n}(q)=f_{n+1}(q)-$ $a_{1}\left(q^{n}, q\right) f_{n}(q)$ then it $P f=0$ implies that $Q g=0$. Conversely, the system $P f=0$ is equivalent to the system of equations

$$
\left\{\begin{array}{l}
f(u q, q)-a_{1}(u, q) f(u, q)=g(u, q) \\
Q g(u, q)=0
\end{array}\right.
$$

Now, by induction we have

$$
g(u, q)=\sum_{k=0}^{\infty} \psi_{k}(n, q) u^{k}
$$

where $\psi_{k}(n, q) \in K[n]$ are polynomials of degree at most $d-1$. Set $f(u, q)=\sum_{k=0}^{\infty} \phi_{k}(n, q) u^{k}$ where $\phi_{k}(n, q) \in K[n]$ are polynomials of degree at most $d$ in the first Equation (17). Both sides are elements of $K[[u]][n]$. The vanishing of each coefficient of $u^{k} n^{i}$ computes the $\phi_{k}$ in terms of the $\psi_{k}$ by a rank 1 linear system of equations. It follows that we can find a $d$-dimensional linear space of WKB series solutions of $P f=0$. This concludes the proof of Theorem 1.2.

\subsection{The regular-singular non-resonant case}

For completeness, we give a short proof of Theorem 1.2 in the case of a non-degenerate regularsingular operator. Recall from Section 1.3 that a regular-singular $q$-difference operator has the form

$$
P=\sum_{i=0}^{d} a_{i}(M, q) L^{i}
$$

where $a_{i}(M, q) \in K\left[\left[M^{1 / r}\right]\right]$ for all $i$ and $a_{0}(0, q) a_{d}(0, q) \neq 0$. The characteristic polynomial $\chi(x, M, q)$ of of a regular-singular operator $P$ is given by

$$
\chi(x, M, q):=\sum_{i=0}^{d} a_{i}(M, q) x^{i}
$$

The eigenvalues of $P$ are the roots of the equation $\chi(x, M, q)=0$. Since $K$ is algebraically closed, Puiseux's theorem implies that the eigenvalues of $P$ are elements of the field $K\{\{q\}\}$; see [Wal78]. 
In other words, a regular-singular operator $P$ has $d$ eigenvalues $\lambda_{i}(M, q) \in K\left(\left(M^{1 / r^{\prime}}\right)\right)$ for some $r^{\prime}$. After we replace $r$ by $r^{\prime}$, we assume $r^{\prime}=r$ below. We say that a regular singular operator $P$ is non-degenerate if its eigenvalues satisfy the non-resonanse condition that $\lambda_{j}(0, q) / \lambda_{i}(0, q) \neq q^{k}$ for all $i \neq j$ and all $k$.

Suppose that $P$ is a non-degenerate regular-singular operator as above. Consider Equation

$$
a_{d}(u, q) f\left(u q^{d}, q\right)+\cdots+a_{0}(u, q) f(u, q)=0
$$

We will show that the general solution to (20) is a WKB sum. Without loss of generality we assume $r=1$. We will construct a basis of solutions of the Equation (20) of the form

$$
f(u, q)=\lambda(q)^{n} \sum_{k=0}^{\infty} \phi_{k}(q) u^{k}
$$

that satisfy $\phi_{0}(q)=1$. Here $n=\log u / \log q$. The point is that although $f(u, q) \notin K[[u]]$, the ratio $f\left(u q^{j}, q\right) / \lambda(q)^{n} \in K[[u]]$ for every $j$. This follows from Equation (9). Substitute (21) into (6) and divide by $\lambda(q)^{n}$. The result is an equation in $K[[u]]$. The vanishing of the coefficient of $u^{0}$ gives

$$
\sum_{j=0}^{d} a_{j}(0, q) \lambda(q)^{j}=0
$$

i.e., $x=\lambda(q)$ is a root of the equation $\chi(x, 0, q)=0$. The vanishing of the coefficient of $u^{k}$ for $k>0$ is the condition

$$
\phi_{k}(q) c_{0, k}(q)+\sum_{i=1}^{k} \phi_{k-i}(q) c_{i, k-i}(q)=0
$$

where

$$
c_{i, j}(q)=\left.(d / d u)^{i} \chi(x, u, q)\right|_{(x, u)=\left(q^{j} \lambda(q), 0\right)} \cdot
$$

Using the initial condition $\phi_{0}=1$, it follows inductively that

$$
\psi_{k}(q):=\phi_{k}(q) \prod_{j=1}^{k} c_{0, j}(q)
$$

is a homogeneous polynomial of total degree $k$ in the variables $c_{i, j}(q)$, where the degree of $c_{i, j}(q)$ is $i+j$. For example, we have:

$$
\begin{aligned}
\psi_{1}= & -c_{1,0} \\
\psi_{2}= & c_{1,0} c_{1,1}-c_{0,1} c_{2,0} \\
\psi_{3}= & -c_{1,0} c_{1,1} c_{1,2}+c_{0,1} c_{1,2} c_{2,0}+c_{0,2} c_{1,0} c_{2,1}-c_{0,1} c_{0,2} c_{3,0} \\
\psi_{4}= & c_{1,0} c_{1,1} c_{1,2} c_{1,3}-c_{0,1} c_{1,2} c_{1,3} c_{2,0}-c_{0,2} c_{1,0} c_{1,3} c_{2,1}-c_{0,3} c_{1,0} c_{1,1} c_{2,2}+c_{0,1} c_{0,3} c_{2,0} c_{2,2} \\
& +c_{0,1} c_{0,2} c_{1,3} c_{3,0}+c_{0,2} c_{0,3} c_{1,0} c_{3,1}-c_{0,1} c_{0,2} c_{0,3} c_{4,0} \\
\psi_{5}= & -c_{1,0} c_{1,1} c_{1,2} c_{1,3} c_{1,4}+c_{0,1} c_{1,2} c_{1,3} c_{1,4} c_{2,0}+c_{0,2} c_{1,0} c_{1,3} c_{1,4} c_{2,1}+c_{0,3} c_{1,0} c_{1,1} c_{1,4} c_{2,2} \\
& -c_{0,1} c_{0,3} c_{1,4} c_{2,0} c_{2,2}+c_{0,4} c_{1,0} c_{1,1} c_{1,2} c_{2,3}-c_{0,1} c_{0,4} c_{1,2} c_{2,0} c_{2,3}-c_{0,2} c_{0,4} c_{1,0} c_{2,1} c_{2,3} \\
& -c_{0,1} c_{0,2} c_{1,3} c_{1,4} c_{3,0}+c_{0,1} c_{0,2} c_{0,4} c_{2,3} c_{3,0}-c_{0,2} c_{0,3} c_{1,0} c_{1,4} c_{3,1}-c_{0,3} c_{0,4} c_{1,0} c_{1,1} c_{3,2} \\
& +c_{0,1} c_{0,3} c_{0,4} c_{2,0} c_{3,2}+c_{0,1} c_{0,2} c_{0,3} c_{1,4} c_{4,0}+c_{0,2} c_{0,3} c_{0,4} c_{1,0} c_{4,1}-c_{0,1} c_{0,2} c_{0,3} c_{0,4} c_{5,0}
\end{aligned}
$$

THE ELECTROnic Journal of COMBinatorics 18(2) (2011), \#P4 
Note that $c_{0, k}=\chi\left(q^{k} \lambda(q), 0, q\right) \neq 0$ for all $k$ due to the non-degeneracy of (20). It remains to show that there exists $c$ such that $\delta\left(\phi_{k}(q)\right) \geq c k$ for all $k$. To prove this, observe that

$$
c_{0, k}(q)=\sum_{j=0}^{d} a_{j}(0, q)\left(q^{k} \lambda(q)\right)^{j}
$$

satisfies that

$$
\delta\left(c_{0, k}\right)=\min _{0 \leq j \leq d}\left\{\delta\left(a_{j}(0, q) \lambda(q)^{j}\right)+k j\right\}
$$

is attained at least twice. Since $a_{0}(0, q) \neq 0$, it follows that for large $k$, the above minimum is attained at $j=0$. In other words, we have

$$
\delta\left(c_{0, k}\right)=c:=\delta\left(a_{0}(0, q)\right) .
$$

Thus,

$$
\delta\left(\prod_{i=1}^{k} c_{0, i}(q)\right) \geq c k+c^{\prime}
$$

for all $k$ large enough. On the other hand, $\delta\left(c_{i, j}(q)\right) \geq 0$ for all $i, j$. The above formulas imply that $\delta\left(\phi_{k}(q)\right) \geq c^{\prime} k$ for all $k$. Thus, for every eigenvalue $\lambda(0, q)$ of $P$ there is a unique WKB solution (21) of Equation (20). Since there are $d$ distinct eigenvalues, the constructed solutions form a basis for the vector space of solutions of (20). This gives a proof of Theorem 1.2 in the non-degenerate regular-singular case.

Remark 2.4. In the case of regular-singular linear differential equations, expressions of the form (22) appear. However, one needs to estimate the numerator of those expressions, as well as the denominator. See for example, [GG06] and references therein. This explains why the WKB theory of $q$-difference equations at $q=0$ is much simpler than the corresponding theory at $q=1$.

\section{Proof of Theorem 1.4}

\subsection{Generalized power sums}

An important special case of Theorem 1.1 is the case of a linear recursion with constant coefficients. In this rather trivial case, for every $n, f_{n}(q)$ is a constant function of $q$, so the degree is easy to compute.

Generalized power sums play a key role to the SML theorem. For a detailed discussion, see [vdP89] and also [EvdPSW03]. Recall that a generalized power sum $a_{n}$ for $n=0,1,2, \ldots$ is an expression of the form

$$
a_{n}=\sum_{i=1}^{m} A_{i}(n) \alpha_{i}^{n}
$$

with roots $\alpha_{i}, 1 \leq i \leq m$, distinct nonzero quantities, and coefficients $A_{i}(n)$ polynomials respectively of degree $n(i)-1$ for positive integers $n(i), 1 \leq i \leq m$. The generalized power sum $a_{n}$ is said to have order

$$
d=\sum_{i=1}^{m} n(i)
$$

THE ELECTRONiC JOURNAL OF COMBINATORICS 18(2) (2011), \#P4 
and satisfies a linear recursion with constant coefficients of the form

$$
a_{n+d}=s_{1} a_{n+d-1}+\cdots+s_{d} a_{n}
$$

where

$$
s(x)=\prod_{i=1}^{m}\left(1-\alpha_{i} x\right)^{n(i)}=1-s_{1} x-\ldots s_{d} x^{d} .
$$

It is well known that a sequence satisfies a linear recursion with constant coefficients if and only if it is a generalized power sum. The LMS theorem concerns the zeros of a generalized power sum.

Theorem 3.1. [Sko35, Mah35, Lec53] The zero set of a generalized power sum is the union of a finite set and a finite set of arithmetic progressions.

\subsection{Proof of theorem 1.4}

Consider a formal WKB series

$$
f_{\tau, n}(q)=q^{n^{2} \gamma+\eta n} \mu^{n} \sum_{j, k \geq 0} c_{\tau, j, k}(n) q^{\frac{j+n k}{r}}
$$

where $\eta=\delta(\lambda(q)), \mu=\operatorname{lt}(\lambda(q)) \in \overline{\mathbb{Q}}$ and $c_{\tau, j, k}(n)$ are generalized power sums with $r$ th roots of 1 . Fix a sequence $f_{n}(q)$ given by a finite $\mathbb{Q}\left(\left(q^{1 / r}\right)\right)$-linear combination of formal WKB series $f_{\tau_{i}}(u, q)$. It follows that

$$
f_{n}(q)=\sum_{i \in I} c_{i}(q) f_{\tau_{i}, n}(q)=\sum_{i \in I} q^{n^{2} \gamma_{i}+\eta_{i} n} \mu_{i}^{n} \sum_{j, k \geq 0} c_{i, j, k}(n) q^{\frac{j+n k}{r}}
$$

where $I$ is a finite set, $c_{i}(q) \in \overline{\mathbb{Q}}\left(\left(q^{1 / r}\right)\right)$ are non-zero and $\left(\tau_{i}, \lambda_{i}\right)$ are pairwise distinct. Consider the subset $I^{\prime}$ of $I$ where the minimum

$$
\min _{i \in I}\left\{\gamma_{i}\right\}
$$

is achieved.

Case 1. If $I^{\prime}$ consists of a single element $i_{0}$ and $c_{i_{0}, j_{0}, k_{0}}(n)$ is not identically zero, then let us concentrate on the following part of $f_{n}(q)$ :

$$
f_{n}(q)=q^{n^{2} \gamma_{i_{0}}+\eta_{i_{0}} n} \mu_{i_{0}}^{n} c_{i_{0}, j_{0}, k_{0}}(n) q^{\frac{j_{0}+n k_{0}}{r}}+\ldots
$$

The LMS theorem concludes that there is a finite set of full arithmetic progressions that cover the set of natural numbers with finite complement, such that the restriction of the degree of $f_{n}(q)$ to each full arithmetic progression (with the exception of a finite set) is given by $n^{2} \gamma_{i_{0}}+\eta_{i_{0}} n+\frac{j_{0}+n k_{0}}{r}$ and the leading term is given by $\mu_{i_{0}}^{n} c_{i_{0}, j_{0}, k_{0}}(n)$. When $c_{i_{0}, j_{0}, k_{0}}(n)$ vanishes consider the next term in the series (24) and repeat the above argument. After finitely many repetitions the process will stop since $f_{\tau_{i}, n}(q)$ are $\overline{\mathbb{Q}}\left(\left(q^{1 / r}\right)\right)$-linearly independent.

Case 2. If $I^{\prime}$ consists of more than one element, without loss of generality, assume that $c_{i, 0,0}(n)$ are not identically zero for $i \in I^{\prime}$. Consider the subset $I^{\prime \prime}$ of $I^{\prime}$ where the maximum

$$
\max _{i \in I^{\prime}}\left\{\eta_{i}\right\}
$$

is achieved. 
Case 2.1. $I^{\prime \prime}$ consists of a single element $i_{0}$. Then, it follows that

$$
f_{n}(q)=q^{n^{2} \gamma_{i_{0}}+\eta_{i_{0}} n} \mu_{i_{0}}^{n} c_{i_{0}, 0,0}(n)+\ldots
$$

and the proof proceeds as in Case 1.

Case 2.2. $I^{\prime \prime}$ consists of more than a single element $i_{0}$. In that case, it follows that

$$
f_{n}(q)=q^{n^{2} \gamma_{i_{0}}+\eta_{i_{0}} n} \sum_{i \in I^{\prime \prime}} \mu_{i}^{n} c_{i, 0,0}(n)+\ldots
$$

The leading term of $f_{n}(q)$ is a generalized power sum and the proof proceeds as in Case 1 .

\section{Invariants of $q$-holonomic sequences}

\subsection{Synopsis of invariants}

In this section, which is independent of the results of our paper, we summarize various invariants of a a $q$-holonomic sequence. Some of these invariants were announced in [Gar04, Gar11, Gar]. In this section $f$ denotes a $q$-holonomic sequence $f_{n}(q) \in \mathbb{Q}(q)$ that satisfies Equation (1) where $a_{i}(M, q) \in \mathbb{Q}(M, q)$. Here is a summary of invariants of $f$.

(a) The annihilator polynomial $P_{f}$

(b) The 2 and 3-dimensional Newton polytopes.

(c) The characteristic curve $\mathrm{ch}_{f}$.

(d) The tropical curve $\mathcal{T}_{f}$.

(e) The degree and leading term of $f$.

As was explained in [Gar], these invariants fit well together and read information of the $q$ holonomic sequence $f$. For completeness, we will present these ideas here, too.

\subsection{The annihilating polynomial of a $q$-holonomic sequence}

In this section we discuss the annihilating polynomial of a $q$-holonomic sequence $f$. Informally, it is the minimal order recursion relation (homogeneous or not) that $f$ satisfies. This was studied in [Gar04] and [Gar]. We will call an operator $P$ given by (5) with $r=1$ reduced if $a_{i}(M, q) \in \mathbb{Z}[M, q]$ are coprime. Let

$$
\mathcal{D}^{\prime}=\mathbb{Q}(M, q)\langle L\rangle /(L M-q M L)
$$

denote the localized $q$-Weyl algebra.

First, we define the homogeneous annihilator $P_{f}$ of $f$. Consider the $\mathcal{D}^{\prime}$-module (nonzero, since $f$ is $q$-holonomic)

$$
M_{f}=\left\{P \in \mathcal{D}^{\prime} \mid P f=0\right\}
$$

and its unique monic generator $P^{\prime} \in \mathcal{D}^{\prime}$. After multiplying by the common denominator of the coefficients of $P^{\prime}$ with respect to the powers of $L$, we obtain a unique reduced generator $P_{f}$. 
Next, we define the non-homogeneous annihilator $\left(P_{f}, b_{f}\right)$ of $f$. Consider the $\mathcal{D}^{\prime}$-module

$$
M_{f}^{n h}=\left\{(P, b) \in \mathcal{D}^{\prime} \times \mathbb{Q}(M, q) \mid P f=b\right\}
$$

$M_{f}^{n h}$ has a unique generator of the form $\left(P^{\prime}, \epsilon\right)$ where $P^{\prime} \in \mathcal{D}^{\prime}$ is monic (with respect to $L$ ) and $\epsilon \in\{0,1\}$. After multiplying by the common denominator of the coefficients of $P^{\prime}$ with respect

to the powers of $L$, we obtain a unique generator of the form $\left(P_{f}^{n h}, b_{f}\right)$ where $P_{f}^{n h}$ is reduced and $b_{f} \in \mathbb{Z}[M, q]$.

Definition 4.1. We call $P_{f}$ and $\left(P_{f}^{n h}, b_{f}\right)$ the homogeneous and the non-homogeneous annihilator of the $q$-holonomic sequence $f$, respectively.

In $\left[\right.$ Gar, Lem.1.4] we show how $P_{f}$ determines $\left(P_{f}^{n h}, b_{f}\right)$ and vice-versa.

\subsection{The characteristic variety of a $q$-holonomic sequence}

The characteristic curve of a $q$-holonomic sequence $f$ is the zero set

$$
\operatorname{ch}_{f}=\left\{(L, M) \in\left(\mathbb{C}^{*}\right)^{2} \mid P_{f}(L, M, 1)=0\right\}
$$

In case $f$ is the colored Jones polynomial of a knot $K$, the AJ Conjecture of [Gar11] identifies the characteristic curve with a geometric knot invariant, namely the moduli space of $\mathrm{SL}(2, \mathbb{C})$ representations of the knot complement.

\subsection{The Newton polytope of a $q$-holonomic sequence}

In this section we consider the 3-dimensional and the 2-dimensional Newton polytope of a $q$ holonomic sequence $f$. Let us write the annihilating polynomial $P_{f}$ of $f$ in terms of monomials:

$$
P_{f}=\sum_{(i, j, k) \in \mathcal{A}} a_{i, j, k} q^{k} M^{j} L^{i}
$$

where $a_{i, j, k} \in \mathbb{Q}$ and the sum is over a finite subset $\mathcal{A}$ of $\mathbb{N}^{3}$ which depends on $f$.

Definition 4.2. (a) The 3-dimensional Newton polytope $\mathcal{N}_{3}\left(P_{f}\right)$ of $f$ is the convex hull $\mathcal{N}_{3}\left(P_{f}\right)$ of $\mathcal{A}$.

(b) The 2-dimensional Newton polytope $\mathcal{N}_{2}\left(P_{f}\right)$ of $f$ is in $\mathbb{R}^{3}$ is the projection of $\mathcal{N}_{3}\left(P_{f}\right)$ on $\mathbb{R}^{2}$ under the map $(x, y, z) \mapsto(x, y)$.

The width of $\mathcal{N}_{3}\left(P_{f}\right)$ with respect to $L$ is the degree of a minimal order recursion relation for $f$, and it is a measure of the complexity of $f$. Likewise, the width of $\mathcal{N}_{3}\left(P_{f}\right)$ with respect to $M$ is a measure of the complexity of the coefficients of a recursion relation of $f$. Knowing (or guessing) $\mathcal{N}_{3}\left(P_{f}\right)$ given some terms in $f$ has applications in Quantum Topology.

Generically one expects that the Newton polygon of the polynomial $P_{f}(x, y, 1)$ coincides with $\mathcal{N}_{2}\left(P_{f}\right)$.

Lemma 4.3. The lower convex hull of $\mathcal{N}_{2}\left(P_{f}\right)$ coincides with the Newton polygon $N\left(P_{f}\right)$ of $P_{f}$ from Section 1.3. 


\subsection{The tropical curve of a $q$-holonomic sequence}

In this section, we assign a tropical curve $\mathcal{T}_{f}$ to a $q$-holonomic sequence $f$, following [Gar]. For a leisure introduction to tropical geometry, see [RGST05, SS09, Stu02, Gar] and references therein. The main idea is to think of an operator in $M, L$ with coefficients in $\mathbb{Q}(q)$ as a $q$-parameter family of polynomials in two commuting variables. More precisely, the coefficients of the annihilating polynomial $P_{f}$ given by (28) define a function

$$
F: \mathbb{R}^{2} \longrightarrow \mathbb{R}, \quad F(x, y)=\min _{(i, j, k) \in \mathcal{A}}\{i x+j y+k\}
$$

$F$ is a piecewise linear convex function. The locus of the points in $\mathbb{R}^{2}$ where $F$ is not differentiable is the tropical curve $\mathcal{T}_{f}$ of $f$. By definition, $\mathcal{T}_{f}$ is the locus of points $(x, y) \in \mathbb{R}^{2}$ where the minimum in (29) is achieved at least twice. It is well-known that $\mathcal{T}_{f}$ consists of a finite collection of line segments with rational vertices, and a finite collection of rays with rational slopes, together with a set of multiplicities that satisfy a balancing condition. Abstractly, a tropical curve is a balanced rational graph, and vice-versa; see [RGST05, Thm.3.6].

There is a duality between Newton polytopes and tropical curves. Indeed, the projection of the lower hull of $\mathcal{N}_{3}\left(P_{f}\right)$ gives a Newton subdivision of $\mathcal{N}_{2}\left(P_{f}\right)$. The tropical curve $\mathcal{T}_{f}$ is dual to the Newton subdivision of $\mathcal{N}_{2}\left(P_{f}\right)$. For drawings of tropical curves of $q$-holonomic sequences of geometric origin, see [Gar].

\subsection{A tropical equation for the degree of a $q$-holonomic sequence}

In this section we explain the relation between the degree $\delta(n)=\delta\left(f_{n}(q)\right)$ of a $q$-holonomic sequence $f$ and its tropical curve $\mathcal{T}_{f}$, following [Gar]. Since $f$ is annihilated by $P_{f}$ (given by $(28)$ ), it follows that for all natural numbers $n$ we have:

$$
\sum_{(i, j, k) \in \mathcal{A}} a_{i, j, k} q^{k+j n} f_{n+i}(q)=0 .
$$

Divide both sides by $f_{n}(q)$ and look at the degree with respect to $q$. It follows that for all $n$, $\delta(n)$ satisfies the following tropical equation: the minimum

$$
\min _{(i, j, k) \in \mathcal{A}}\{\delta(n+i)-\delta(n)+j n+k\}
$$

is achieved at least twice. Although (30) may have non quasi-polynomial solutions, Theorem 1.1 suggests to look for quadratic quasi-polynomial solutions of (30). It turns out that those solutions $\delta(n)$ are determined by the tropical curve $\mathcal{T}_{f}$ as follows. For $n$ in an arithmetic progression minus finitely many values, we have:

$$
\delta(n)=\frac{c_{2}}{2} n^{2}+c_{1} n+c_{0}
$$

Proposition 4.4. $\left(c_{1}, c_{2}\right)$ satisfy the system of equations

$$
\begin{aligned}
c_{2} i+j & =c_{2} i^{\prime}+j^{\prime} \\
\frac{1}{2} c_{2} i^{2}+c_{1} i+k & =\frac{1}{2} c_{2} i^{\prime 2}+c_{1} i^{\prime}+k^{\prime}
\end{aligned}
$$

for two distinct points $(i, j, k)$ and $\left(i^{\prime}, j^{\prime}, k^{\prime}\right)$ of $\mathcal{A}$. 
Proof: For $n$ in an arithmetic progression minus finitely many values we have: the minimum

$$
\min _{(i, j, k) \in \mathcal{A}}\left\{n\left(c_{2} i+j\right)+\frac{1}{2} c_{2} i^{2}+c_{1} i+k\right\}
$$

is achieved at least twice. It follows that there are two distinct points $(i, j, k)$ and $\left(i^{\prime}, j^{\prime}, k^{\prime}\right)$ of $\mathcal{A}$ such that Equations (32) and (33) hold. Equation (32) involves only $(i, j)$ and $\left(i^{\prime}, j^{\prime}\right)$ and says that $c_{2}$ is the negative of a slope of $N\left(P_{f}\right)$. Likewise Equation (32) involves only $(i, k)$ and $\left(i^{\prime}, k^{\prime}\right)$.

Remark 4.5. It would be interesting to study the solutions to the tropical equation (30) independent of differential Galois theory.

Remark 4.6. Each Equation (32) and (33) describes a tropical curve in $\mathbb{R}^{2}$. However, the system of Equations (32) and (33) is not the intersection of the two tropical curves since the two planes $\mathbb{R}^{2}$ are different projections of $\mathbb{R}^{3}$.

\subsection{Future directions}

$q$-holonomic sequences of several variables $f_{n_{1}, \ldots, n_{r}}(q) \in \mathbb{Z}\left[q^{ \pm 1}\right]$ also appear in Quantum Topology. For example the colored Jones function of a 2-component link, of the $\mathfrak{s l}_{3}$-colored Jones polynomial of a knot is a $q$-holonomic sequence of two variables. Suitably formulated, Theorem 1.1 should extend to $q$-holonomic sequences of many variables. This will be explained in a future publication.

\section{Acknowledgment}

The idea of the present paper was conceived during the New York Conference on Interactions between Hyperbolic Geometry, Quantum Topology and Number Theory in New York in the summer of 2009. An early version of the present paper appeared in the New Zealand Conference on Topological Quantum Field Theory and Knot Homology Theory in January 2010 and a finished version appeared in the Conference in Rutgers in honor of D. Zeilberger's 60th birthday. The author wishes to thank the organizers of the New York Conference, A. Champanerkar, O. Dasbach, E. Kalfagianni, I. Kofman, W. Neumann and N. Stoltzfus, the New Zealand Conference, R. Fenn, D. Gauld and V. Jones and the Rutgers Conference, for their hospitality. In addition, the author wishes to thank E. Croot, T. Dimofte, D. Zagier, D. Zeilberger and J. Yu for stimulating conversations.

\section{References}

[BB92] Jean-Paul Bézivin and Abdelbaki Boutabaa, Sur les équations fonctionelles padiques aux q-différences, Collect. Math. 43 (1992), no. 2, 125-140.

[BR07] Matthias Beck and Sinai Robins, Computing the continuous discretely, Undergraduate Texts in Mathematics, Springer, New York, 2007, Integer-point enumeration in polyhedra. 
[DV08] Lucia Di Vizio, An ultrametric version of the Maillet-Malgrange theorem for nonlinear q-difference equations, Proc. Amer. Math. Soc. 136 (2008), no. 8, 28032814 .

[EvdPSW03] Graham Everest, Alf van der Poorten, Igor Shparlinski, and Thomas Ward, Recurrence sequences, Mathematical Surveys and Monographs, vol. 104, American Mathematical Society, Providence, RI, 2003.

[Gar] Stavros Garoufalidis, Knots and tropical curves, Contemporary Mathematics, in press.

[Gar04] _ On the characteristic and deformation varieties of a knot, Proceedings of the Casson Fest, Geom. Topol. Monogr., vol. 7, Geom. Topol. Publ., Coventry, 2004, pp. 291-309 (electronic).

[Gar11] _ The Jones slopes of a knot, Quantum Topol. 2 (2011), 43-69.

[GG06] Stavros Garoufalidis and Jeffrey S. Geronimo, Asymptotics of q-difference equations, Primes and knots, Contemp. Math., vol. 416, Amer. Math. Soc., Providence, RI, 2006, pp. 83-114.

[GL05] Stavros Garoufalidis and Thang T. Q. Lê, The colored Jones function is qholonomic, Geom. Topol. 9 (2005), 1253-1293 (electronic).

[Hor09] Peter Horn, Factorisierung in schief-polynomringen, Ph.D. thesis, University of Kassel, 2009.

[Lec53] Christer Lech, A note on recurring series, Ark. Mat. 2 (1953), 417-421.

[Mah35] Kurt Mahler, Eine arithmetische eigenschaft der taylor koeffizienten rationaler funktionen, Tech. Report 38, Proc. Akad. Wet. Amsterdam, 1935, 51-60.

[Mar] Thomas Markwig, polymake and tropical.lib, http://www. mathematik. uni-kl.de/ keilen/en/tropical.html.

[PR] Peter Paule and Axel Riese, qZeil Mathematica software, http://www.risc.uni-linz.ac.at.

[PR97] _ A Mathematica q-analogue of Zeilberger's algorithm based on an algebraically motivated approach to q-hypergeometric telescoping, Special functions, $q$-series and related topics (Toronto, ON, 1995), Fields Inst. Commun., vol. 14, Amer. Math. Soc., Providence, RI, 1997, pp. 179-210.

[PWZ96] Marko Petkovšek, Herbert S. Wilf, and Doron Zeilberger, $A=B$, A K Peters Ltd., Wellesley, MA, 1996, With a foreword by Donald E. Knuth, With a separately available computer disk.

[RGST05] Jürgen Richter-Gebert, Bernd Sturmfels, and Thorsten Theobald, First steps in tropical geometry, Idempotent mathematics and mathematical physics, Contemp. Math., vol. 377, Amer. Math. Soc., Providence, RI, 2005, pp. 289-317.

[Sko35] Th. Skolem, Ein verfahren zur behandlung gewisser exponentialer gleichungen, Tech. report, Comptes Rendus du 8e congrès des mathématiciens scandinaves, Lund Hakan Ohlssons Boktryckeri, 1935, 163-188.

[SS09] David Speyer and Bernd Sturmfels, Tropical mathematics, Math. Mag. 82 (2009), no. $3,163-173$. 
[Sta97] Richard P. Stanley, Enumerative combinatorics. Vol. 1, Cambridge Studies in Advanced Mathematics, vol. 49, Cambridge University Press, Cambridge, 1997, With a foreword by Gian-Carlo Rota, Corrected reprint of the 1986 original.

[Stu02] Bernd Sturmfels, Solving systems of polynomial equations, CBMS Regional Conference Series in Mathematics, vol. 97, Published for the Conference Board of the Mathematical Sciences, Washington, DC, 2002.

[vdP89] A. J. van der Poorten, Some facts that should be better known, especially about rational functions, Number theory and applications (Banff, AB, 1988), NATO Adv. Sci. Inst. Ser. C Math. Phys. Sci., vol. 265, Kluwer Acad. Publ., Dordrecht, 1989, pp. 497-528.

[vdPS03] Marius van der Put and Michael F. Singer, Galois theory of linear differential equations, Grundlehren der Mathematischen Wissenschaften [Fundamental Principles of Mathematical Sciences], vol. 328, Springer-Verlag, Berlin, 2003.

[Wal78] Robert J. Walker, Algebraic curves, Springer-Verlag, New York, 1978, Reprint of the 1950 edition.

[Wit] Edward Witten, Fivebranes and knots, arXiv:1101.3216, Preprint 2011.

[WZ92] Herbert S. Wilf and Doron Zeilberger, An algorithmic proof theory for hypergeometric (ordinary and "q") multisum/integral identities, Invent. Math. 108 (1992), no. 3, 575-633.

[Zei90] Doron Zeilberger, A holonomic systems approach to special functions identities, J. Comput. Appl. Math. 32 (1990), no. 3, 321-368. 